\title{
THE SECOND ORDER OF ADS FOR REVERSE PARABOLIC BOUNDARY VALUE PROBLEM WITH INTEGRAL CONDITION
}

\author{
CHARYYAR ASHYRALYYEV
}

\begin{abstract}
Finite difference method to solve nonlocal reverse parabolic (RP) problem with integral condition are applied. The second order of accuracy difference scheme (ADS) for approximation of RP problem is proposed. Stability estimates (SEs) for solutions of this difference scheme (DS) are proved. Later, we propose the second order of ADS for approximation of multidimensional RP boundary value problem (BVP) with first kind boundary condition (BC) and establish SEs for its solution. Numerical illustration for simple test problems is given.
\end{abstract}

\section{Preliminaries}

Nonlocal BVPs for parabolic equations are used to model of biological, physical, system engineering and sociological processes and have been studied by many authors (see $[1,2,3,4,5,6,7,8,9,10,11,12,13,15,16,17,18,19,20,23,24$, 25, 26] and bibliography therein).

RP equation arise in many applications such as fluid dynamics, plasma physics, study of propagation of an electron beam through the solar corona [13, 18, 19, 23, 24]. In paper [17], computation of mean field equilibria in economics is carried out by using model with RP equation.

Well-posedness of non classical BVPs for RP equation with various boundary conditions and DSs for approximations were investigated in $[2,3,4,6,7,9,10]$. In paper [2], the authors established well-posedness of multipoint nonlocal BVPs for RP equations. In [3, 4], first and second orders DSs for approximate solution of multipoint nonlocal BVPs for RP equations were studied. In [8, 9], second order DSs for approximate solution of multipoint nonlocal problems for multidimensional RP equations with Dirichlet and Neumann boundary conditions, respectively.

In paper [6], the RP problem

2010 Mathematics Subject Classification. 39A14, 65J22.

Key words and phrases. Difference scheme, well-posedness, integral condition, stability estimates, reverse parabolic problem. 


$$
\left\{\begin{array}{l}
u_{t}(t)-A u(t)=f(t), 0 \leq t \leq 1 \\
u(1)=\int_{0}^{1} \mu(s) u(s) d s+\varphi
\end{array}\right.
$$

with integral condition and some BVPs to multidimensional RP equation were investigated on well-posedness under assumption $\int_{0}^{1}|\mu(s)| d s \leq 1$ for coefficient function in integral condition.

Here $H$ is an arbitrary Hilbert space, $A$ is self-adjoint positive definite (SAPD) operator in $H, I$ is identity operator, $A>\delta I \quad(\delta>0), \mu:[0,1] \rightarrow R, f:[0,1] \rightarrow$ $H$ are given functions and $\varphi$ is known element is in $H$.

Let $[0,1]_{\tau}=\left\{t_{i} \mid t_{i}=i \tau\right\}$ be the uniform grid space for some fixed natural number $N, \tau=\frac{1}{N}$ and $C_{\tau}(H)=C\left([0,1]_{\tau}, H\right)$ be the linear space of the corresponding grid functions $v^{\tau}=\left\{v_{i}\right\}_{i=1}^{i=N}$ with values $v_{i}=v\left(t_{i}\right)$ in $H$. By $C_{\tau}(H), C_{\tau}^{\alpha}(H)=C^{\alpha}\left([0,1]_{\tau}, H\right)$, and $C_{1}^{\alpha}(H)=C_{1}^{\alpha}\left([0,1]_{\tau}, H\right)$, we denote Banach spaces of $v^{\tau}$ functions with the corresponding norms

$$
\begin{aligned}
\left\|v^{\tau}\right\|_{C_{\tau}(H)} & =\max _{1 \leq i \leq N}\left\|v_{i}\right\|_{H}, \\
\left\|v^{\tau}\right\|_{C_{\tau}^{\alpha}(H)} & =\left\|v^{\tau}\right\|_{C_{\tau}(H)}+\max _{1 \leq i<i+j \leq N}(j \tau)^{-\alpha}\left\|v_{i+j}-v_{i}\right\|_{H}, \\
\left\|v^{\tau}\right\|_{C_{1}^{\alpha}(H)} & =\left\|v^{\tau}\right\|_{C_{\tau}^{\alpha}(H)}+\max _{1 \leq i<i+j \leq N}(j \tau)^{-\alpha}((N-i) \tau)^{\alpha}\left\|v_{i+j}-v_{i}\right\|_{H} .
\end{aligned}
$$

In [7], stability estimates for solution of first order DS for approximate solution of RP problem with integral condition (1.1) were established under some condition for coefficient function. However, approximation of BVPs for RP equation with integral conditions have not been well-investigated so far. Therefore, the main aim of this paper is to study second order of ADS for approximation of RP problem with nonlocal integral condition (1.1):

$$
\begin{gathered}
\quad \frac{u_{i}-u_{i-1}}{\tau}-A\left(I+\frac{\tau}{2} A\right) u_{i-1}=\left(I+\frac{\tau}{2} A\right) f\left(\left(i-\frac{1}{2}\right) \tau\right), \\
t_{i}=i \tau, \quad 1 \leq i \leq N, \tau=\frac{1}{N}, \\
u_{N}=\sum_{j=1}^{N} \mu\left(t_{j}-\frac{\tau}{2}\right)\left(\frac{u_{j}+u_{j-1}}{2}\right) \tau+\varphi .
\end{gathered}
$$

In future throughout paper will be needed the following assumption:

$$
\sum_{j=1}^{N}\left|\mu\left(t_{j}-\frac{\tau}{2}\right)\right| \tau<1 .
$$

Introduce notations:

$$
\begin{gathered}
F=\left(I+\tau A+\frac{(\tau A)^{2}}{2}\right)^{-1}, \\
\theta_{i}=f\left(\left(i-\frac{1}{2}\right) \tau\right), 1 \leq i \leq N, \theta^{\tau}=\left\{\theta_{i}\right\}_{1}^{N} .
\end{gathered}
$$


Lemma 1.1. The following inequalities hold ([5]):

$$
\begin{aligned}
& \left\|F^{m}-e^{-m \tau A}\right\|_{H \rightarrow H} \leq \frac{M \tau^{2}}{(m \tau)^{2}}, m \geq 1,\left\|(\tau A)^{\gamma} F\left(I+\frac{\tau A}{2}\right)\right\|_{H \rightarrow H} \leq 1, \\
& \gamma \in\{0,1\},\left\|(\tau A)^{\beta} F^{m}\right\|_{H \rightarrow H} \leq \frac{1}{m^{\beta}}, m \geq 1,0 \leq \beta \leq 1, \\
& \left\|\left(I+\frac{\tau A}{2}\right)^{H} F\left(I+\frac{\tau A}{2}\right)\right\|_{H \rightarrow H}^{\leq 1,}\left\|(I+\tau A) F\left(I+\frac{\tau A}{2}\right)\right\|_{H \rightarrow H} \leq 2 .
\end{aligned}
$$

Lemma 1.2. Under the assumption (1.5), for the operator

$$
T_{\tau}=I-\sum_{j=1}^{N} \mu\left(t_{j}-\frac{\tau}{2}\right) \frac{\tau}{2}\left(F^{N-j}+F^{N-j+1}\right)
$$

exists inverse operator

$$
Q_{\tau}=\left(I-\sum_{j=1}^{N} \mu\left(t_{j}-\frac{\tau}{2}\right) \frac{\tau}{2}\left(F^{N-j}+F^{N-j+1}\right)\right)^{-1}
$$

and the inequality

$$
\left\|Q_{\tau}\right\|_{H \rightarrow H} \leq M(\delta)
$$

is satisfied for some constant $M$ which depends only on $\delta$.

Proof. By using definition of function's norm for SAPD operator (see [14]), one can write

$$
\begin{aligned}
& \left\|Q_{\tau}\right\|_{H \rightarrow H} \leq \sup _{\lambda \geq \delta} \frac{1}{\left|1-\sum_{j=1}^{N} \mu\left(t_{j}-\frac{\tau}{2}\right) \tau \frac{1}{\left(1+\tau \lambda+\frac{\tau^{2} \lambda^{2}}{2}\right)^{N-j}} \frac{1}{2}\left(1+\frac{1}{1+\tau \lambda+\frac{\tau^{2} \lambda^{2}}{2}}\right)\right|} \leq M . \\
& \leq \sup _{\lambda \geq \delta} \frac{1}{\left|1-\sum_{j=1}^{N}\right| \mu\left(t_{j}-\frac{\tau}{2}\right)|\tau|} \leq M .
\end{aligned}
$$

The goal of the current paper is to construct stable DS for approximation of RP problem (1.1). So, we will prove SEs for solution of the second order of ADS. Later, we study the the second order of ADS for approximate solution of BVP for RP multidimensional equation and establish stability inequalities for solution of DS. Lasty, by using MATLAB program, we illustrate numerical results in test examples.

\section{Second order of ADS}

Theorem 2.1. Assume that $\theta^{\tau} \in C_{\tau}(H), \varphi \in D(A)$, and (1.5) is satisfied. Then, difference problem (1.3) , (1.4) has solution and stability estimate for its solution

$$
\max _{0 \leq j \leq N}\left\|u_{j}\right\|_{H} \leq M(\delta, \mu)\left(\|\varphi\|_{H}+\left\|\theta^{\tau}\right\|_{C_{\tau}(H)}\right)
$$

is valid, for some positive real constant $M(\delta, \mu)$ which is independent of $\tau, \varphi, \theta^{\tau}$, but depends on $\delta, \mu$. 
Proof. If $u_{N}$ is given, then solution of (1.3) can be defined by

$$
u_{j}=F^{N-j} u_{N}-\sum_{l=j+1}^{N} F^{l-j}\left(I+\tau \frac{A}{2}\right) \theta_{l} \tau, 0 \leq j \leq N-1 .
$$

By virtue (2.2), from (1.4) implies

$$
\begin{aligned}
& u_{N}=\sum_{j=1}^{N} \mu\left(t_{j}-\frac{\tau}{2}\right) \frac{\tau}{2}\left(F^{N-j}+F^{N-j+1}\right) u_{N} \\
& -\sum_{j=1}^{N} \mu\left(t_{j}-\frac{\tau}{2}\right)\left[\sum_{l=j+1}^{N}\left(F^{l-j}+F^{l-j+1}\right)\left(I+\frac{\tau A}{2}\right) \theta_{l} \tau\right] \\
& -\sum_{j=1}^{N} \mu\left(t_{j}-\frac{\tau}{2}\right) F\left(I+\frac{\tau A}{2}\right) \theta_{j} \tau+\varphi .
\end{aligned}
$$

According to the Lemma 2.2, there exists solution of DS (1.3), (1.4) in the form (2.2) with the corresponding value $u_{N}$ :

$$
\begin{aligned}
& u_{N}=-Q_{\tau} \sum_{j=1}^{N} \mu\left(t_{j}-\tau / 2\right)\left[\sum_{l=j+1}^{N}\left(F^{l-j}+F^{l-j+1}\right)\left(I+\frac{\tau}{2} A\right) \theta_{l} \tau\right] \\
& -Q_{\tau} \sum_{j=1}^{N} \mu\left(t_{j}-\frac{\tau}{2}\right) F\left(I+\frac{\tau}{2} A\right) \theta_{j} \tau+Q_{\tau} \varphi .
\end{aligned}
$$

So, difference problem (1.3), (1.4) is uniquely solvable and its solution is defined by $(2.2),(2.4)$.

From (1.5), (2.2), $\tau N=1$ follows

$$
\begin{aligned}
& \left\|u_{j}\right\|_{H} \leq\left\|F^{N-j}\right\|_{H \rightarrow H}\left\|u_{N}\right\|_{H}+\max _{1 \leq l \leq N}\left\|\theta_{l}\right\|_{H} \sum_{l=j+1}^{N}\left\|F^{l-j}\left(I+\frac{\tau}{2} A\right)\right\|_{H \rightarrow H} \tau \\
& \leq M_{1}\left(\left\|u_{N}\right\|_{H}+\left\|\theta^{\tau}\right\|_{C_{\tau}(H)}\right) .
\end{aligned}
$$

Thus,

$$
\max _{0 \leq j \leq N}\left\|u_{j}\right\|_{H} \leq M_{1}\left(\left\|u_{N}\right\|_{H}+\left\|\theta^{\tau}\right\|_{C_{\tau}(H)}\right) .
$$

Under assumption (1.5), by using formula (2.4), we get

$$
\left\|u_{N}\right\|_{H} \leq M_{2}\left(\left\|\theta^{\tau}\right\|_{C_{\tau}(H)}+\|\varphi\|_{H}\right) .
$$

Combining these estimates, one can establish (2.1) and complete proof of statement. 
Theorem 2.2. Assume that $\varphi \in D(A), \theta^{\tau} \in C_{1}^{\alpha}(H)$, and (1.5) is valid. Then, solution of $D S$ (1.3), (1.4) satisfies the coercive $S E$

$$
\begin{aligned}
& \left\|\left\{\frac{u_{i}-u_{i-1}}{\tau}\right\}_{1}^{N}\right\|_{C_{\tau}^{\alpha}(H)}+\left\|\left\{A\left(I+\frac{\tau A}{2}\right) u_{i-1}\right\}_{1}^{N}\right\|_{C_{1}^{\alpha}(H)} \\
& \leq M(\delta, \mu)\left(\frac{1}{\alpha(1-\alpha)}\left\|\theta^{\tau}\right\|_{C_{1}^{\alpha}(H)}+\|A \varphi\|_{H}\right)
\end{aligned}
$$

for some positive real constant $M(\delta, \mu)$ which is independent of $\tau, \varphi, \theta^{\tau}$, but depends on $\delta, \mu$.

Proof. By vitue (2.2), definition of operator $F$, and the following identity

$$
\tau A F\left(I+\frac{\tau}{2} A\right)=I-F
$$

we get

$$
\begin{aligned}
& A\left(I+\frac{\tau}{2} A\right) u_{i-1}=\left(I+\frac{\tau}{2} A\right) F^{N-i+1} A u_{N} \\
& -\sum_{j=i}^{N} A F^{j-i} F\left(I+\frac{\tau}{2} A\right)\left(\theta_{j}-\theta_{i}\right) \tau+\left(F^{N-i+1}-I\right) \theta_{i}
\end{aligned}
$$

for any $1 \leq i \leq N$. By using triangle inequality to (2.7), and by applying estimates (1.8), the definition of norm in $C_{1}^{\alpha}(H)$, we have

$$
\begin{aligned}
& \left\|A\left(I+\frac{\tau A}{2}\right) u_{i-1}\right\|_{H} \leq\left\|F^{N-i+1}\right\|_{H \rightarrow H} \cdot\left\|A u_{N}\right\|_{H} \\
& +\sum_{j=i}^{N} \frac{\left\|\theta^{\tau}\right\|_{C_{1}^{\alpha}(H)} \tau}{(j-i+1)^{1-\alpha}(N-i+1)^{\alpha}}+\left(\left\|F^{N-i+1}\right\|_{H \rightarrow H}+1\right)\left\|\theta^{\tau}\right\|_{C_{1, \tau}^{\alpha}(H)} \\
& \leq\left\|A u_{N}\right\|_{H}+4 \alpha^{-1}\left\|\theta^{\tau}\right\|_{C_{1}^{\alpha}(H)}, i=1, \ldots, N .
\end{aligned}
$$

From definition of $F$ and (2.4) it implies that

$$
\begin{aligned}
& A u_{N}=-Q_{\tau} \sum_{j=1}^{N} \mu\left(t_{j}-\frac{\tau}{2}\right)\left[\sum_{l=j+1}^{N} \tau\left(A F^{l-j}+A F^{l-j+1}\right)\left(I+\frac{\tau}{2} A\right) \theta_{l}\right] \\
& -Q_{\tau} \sum_{j=1}^{N} \mu\left(t_{j}-\frac{\tau}{2}\right) \tau A F\left(I+\frac{\tau}{2} A\right) \theta_{j}+\tau Q A \varphi .
\end{aligned}
$$

So, by (1.5), (1.8), (1.10), (2.9), the definition of $C_{1}^{\alpha}(H)$-norm, one can show

$$
\left\|A u_{N}\right\|_{H} \leq M(\delta, \mu)\left(4 \alpha^{-1}\left\|\theta^{\tau}\right\|_{C_{1}^{\alpha}(H)}+\|A \varphi\|_{H}\right), i=1, \ldots, N .
$$

Therefore, from inequalities (2.8), (2.10) it can be conlcluded that

$$
\left\|\left\{A\left(I+\frac{\tau A}{2}\right) u_{i-1}\right\}_{1}^{N}\right\|_{C_{\tau}(H)} \leq M(\delta, \mu)\left(\alpha^{-1}\left\|\theta^{\tau}\right\|_{C_{1}^{\alpha}(H)}+\|A \varphi\|_{H}\right) .
$$


Now, let us estimate $\left\|\left\{A\left(I+\frac{\tau A}{2}\right) u_{i-1}\right\}_{1}^{N}\right\|_{C_{1}^{\alpha}(H)}$. Let us $N-i+l>2 l$. Then, by virtue (2.2), we have

$$
\begin{aligned}
& A\left(I+\frac{\tau A}{2}\right) u_{i-1}-A\left(I+\frac{\tau A}{2}\right) u_{i-1+l} \\
& =\left(I+\frac{\tau A}{2}\right)\left(F^{N-i+1}-F^{N-i-l+1}\right) A u_{N} \\
& -\sum_{k=i}^{k+2 l-1} \tau A F^{k-i} F\left(I+\frac{\tau A}{2}\right)\left(\theta_{k}-\theta_{i}\right) \\
& -\sum_{k=i+2 l}^{N} \tau A\left(F^{k-i}-F^{k-(i+l)}\right) F\left(I+\frac{\tau A}{2}\right)\left(\theta_{k}-\theta_{i-1}\right) \\
& +\sum_{k+2 l-1} \tau A F^{k-(i+l)} F\left(I+\frac{\tau A}{2}\right)\left(\theta_{k}-\theta_{i+l}\right) \\
& +\left(I-F^{l-1}\right)\left(\theta_{i+l}-\theta_{i}\right)+\left(F^{N-i+1}-F^{N-(i-1+l)}\right) \theta_{i}=\sum_{j=1}^{6} P_{j}(i) .
\end{aligned}
$$

By using (1.8), (1.10), we can show

$$
\left\|P_{1}(i)\right\|_{H} \leq M(\delta, \mu) \frac{(l \tau)^{\alpha}}{((N-i+l) \tau)^{\alpha}}\left(\alpha^{-1}\left\|\theta^{\tau}\right\|_{C_{1}^{\alpha}(H)}+\|A \varphi\|_{H}\right) .
$$

Estimates for $\left\|P_{j}(i)\right\|_{H}(j=2,3,4,5,6)$ were established in the paper [4] by (29)-(33):

$$
\begin{aligned}
& \left\|P_{2}(i)\right\|_{H} \leq \frac{(l \tau)^{\alpha}}{((N-i+l) \tau)^{\alpha}} \frac{4^{\alpha}}{\alpha}\left\|\theta^{\tau}\right\|_{C_{1}^{\alpha}(H)}, \quad\left\|P_{3}(i)\right\|_{H} \leq M \frac{4^{\alpha}}{1-\alpha} \frac{(l \tau)^{\alpha}}{((N-i+l) \tau)^{\alpha}}\left\|\theta^{\tau}\right\|_{C_{1}^{\alpha}(H)}, \\
& \left\|P_{4}(i)\right\|_{H} \leq \frac{3^{\alpha}}{\alpha} \frac{(l \tau)^{\alpha}}{((N-i+l) \tau)^{\alpha}}\left\|\theta^{\tau}\right\|_{C_{1}^{\alpha}(H)}, \quad\left\|P_{5}(i)\right\|_{H} \leq 2^{1+\alpha} \frac{(l \tau)^{\alpha}}{((N-i+l) \tau)^{\alpha}}\left\|\theta^{\tau}\right\|_{C_{1}^{\alpha}(H)}, \\
& \left\|P_{6}(i)\right\|_{H} \leq 3^{\alpha} \frac{(l \tau)^{\alpha}}{((N-i+l) \tau)^{\alpha}}\left\|\theta^{\tau}\right\|_{C_{1}^{\alpha}(H)} .
\end{aligned}
$$

Combining these estimates one can obtain

$$
\begin{aligned}
& \max _{1 \leq i<i+l \leq N} \frac{((N-i+l) \tau)^{\alpha}}{(l \tau)^{\alpha}}\left\|A\left(I+\frac{\tau A}{2}\right) u_{i-1}-A\left(I+\frac{\tau A}{2}\right) u_{i-1+l}\right\|_{H} \\
& \leq M(\delta, \mu)\left(\alpha^{-1}(1-\alpha)^{-1}\left\|\theta^{\tau}\right\|_{C_{1}^{\alpha}(H)}+\|A \varphi\|_{H}\right) .
\end{aligned}
$$

From (2.11) and (2.13) it follows that

$$
\left\|\left\{A\left(I+\frac{\tau A}{2}\right) u_{i-1}\right\}_{1}^{N}\right\|_{C_{1}^{\alpha}(H)} \leq M(\delta, \mu)\left(\alpha^{-1}(1-\alpha)^{-1}\left\|\theta^{\tau}\right\|_{C_{1}^{\alpha}(H)}+\|A \varphi\|_{H}\right) .
$$

Finally, estimate (2.5) implies from (1.3), triangle inequality and estimate (2.14). 


\section{DS for RP multidimensional problem}

Let $\Omega=(0, l) \times(0, l) \times \ldots \times(0, l)$ be open cube in $R^{n}, S=\partial \Omega$,

$\bar{\Omega}=\Omega \cup S$, and $a_{r}: \Omega \rightarrow R, \varphi: \bar{\Omega} \rightarrow R, \mu:[0,1] \rightarrow R, f:(0,1) \times \Omega \rightarrow R$ be given functions, $\sigma$ be known positive real number. In addition, $\forall r=1, \ldots, n$, $\forall x=\left(x_{1}, . ., x_{n}\right) \in \Omega, a_{r}(x) \geq a_{0}>0$.

In the work [6], BVP for RP multidimensional equation with integral and first kind of $\mathrm{BCs}$

$$
\left\{\begin{array}{l}
u_{t}(t, x)+\sum_{r=1}^{n}\left(a_{r}(x) u_{x_{r}}(t, x)\right) x_{r}-\sigma u(t, x)=f(t, x), \\
t \in(0,1), x \in \Omega \\
u(1, x)=\int_{0}^{1} \mu(s) u(s, x) d s+\varphi(x), x \in \bar{\Omega} \\
u(t, x)=0, x \in S, t \in[0,1]
\end{array}\right.
$$

was investigated on well-posedness.

Now, we will construct the second order of ADS to solve BVP (3.1).

Let us denote by

$$
\begin{aligned}
\widetilde{\Omega}_{h} & =\left\{x_{m}=\left(h_{1} m_{1}, \cdots, h_{n} m_{n}\right) ; m=\left(m_{1}, \cdots, m_{n}\right),\right. \\
m_{r} & \left.=0, \cdots, N_{r}, h_{r} N_{r}=l, r=1, \cdots, n\right\}
\end{aligned}
$$

space of grid points and $\Omega_{h}=\Omega \cap \widetilde{\Omega}_{h}, S_{h}=\widetilde{\Omega}_{h} \cap S$, and by $A_{h}^{x}$ the operator $A_{h}^{x} u^{h}(x)=-\sum_{r=1}^{n}\left(a_{r}(x) u_{\bar{x}_{r}}^{h}(x)\right)_{x_{r}, j_{r}}+\sigma u^{h}(x)$ acting in the space of grid functions $u^{h}(x)$ which satisfies the condition $u^{h}(x)=0$ on $x \in S_{h}$.

By using notation $A_{h}^{x}$, the problem (3.1) reduces to the following nonlocal BVP for an infinite system of ordinary differential equations with integral condition

$$
\left\{\begin{array}{l}
\frac{d}{d t} u^{h}(t, x)-A_{h}^{x} u^{h}(t, x)=f^{h}(t, x), t \in(0,1), x \in \widetilde{\Omega}_{h}, \\
u^{h}(1, x)=\int_{0}^{1} \mu(s) u^{h}(s, x) d s+\varphi^{h}(x), x \in \widetilde{\Omega}_{h} .
\end{array}\right.
$$

In sequel, BVP (3.1) is replaced by the the second order of ADS

$$
\left\{\begin{array}{l}
\frac{u_{k}^{h}(x)-u_{k-1}^{h}(x)}{\tau}-A_{h}^{x} B_{h}^{x} u_{k-1}^{h}(x)=\psi_{k}(x), \\
\psi_{k}(x)=B_{h}^{x} f^{h}\left(t_{k-\frac{\tau}{2}}, x\right), B_{h}^{x}=I+\frac{\tau A_{h}^{x}}{2}, \\
t_{k}=k \tau, k=1, \ldots, N, N \tau=1, x \in \widetilde{\Omega}_{h}, \\
u_{N}^{h}(x)=\sum_{j=1}^{N} \frac{\mu\left(t_{j}-\frac{\tau}{2}\right) \tau}{2}\left[u_{j}^{h}(x)+u_{j-1}^{h}(x)\right]+\varphi^{h}(x), x \in \widetilde{\Omega}_{h} .
\end{array}\right.
$$


We denote by $L_{2 h}$ and $W_{2 h}^{2}$ spaces of the grid functions $\varphi^{h}(x)$ defined on grid space $\widetilde{\Omega}_{h}$, equipped with the appropriate norms

$$
\begin{aligned}
\left\|\varphi^{h}\right\|_{L_{2 h}} & =\left(\sum_{x \in \widetilde{\Omega}_{h}}\left|\varphi^{h}(x)\right|^{2} h_{1} \cdots h_{n}\right)^{1 / 2} \\
\left\|\varphi^{h}\right\|_{W_{2 h}^{2}} & =\left(\sum_{x \in \widetilde{\Omega}_{h}} \sum_{r=1}^{n}\left|\left(\varphi^{h}(x)\right)_{x_{r} \overline{x_{r}}, m_{r}}\right|^{2} h_{1} \cdots h_{n}\right)^{1 / 2} .
\end{aligned}
$$

Throughout this section, let $|h|=\left(\sum_{r=1}^{n} h_{r}^{2}\right)^{\frac{1}{2}}$ and $\tau$ be small positive real numbers.

Theorem 3.1. For the solution of $D S$ (3.3) the $S E$

$$
\left\|\left\{u_{k}^{h}\right\}_{1}^{N}\right\|_{\mathcal{C}_{\tau}\left(L_{2 h}\right)} \leq M(\delta, \mu)\left[\left\|\varphi^{h}\right\|_{L_{2 h}}+\left\|\left\{\psi_{k}^{h}\right\}_{1}^{N}\right\|_{\mathcal{C}_{\tau}\left(L_{2 h}\right)}\right]
$$

is fulfilled, where $M(\delta, \mu)$ is independent of $\tau, \varphi^{h}(x)$, and $\left\{\psi_{k}^{h}\right\}_{1}^{N}$.

The proof of Theorem 3.1. is based on estimate (2.1), assumption (1.5), and the following theorem on the coercivity stability property for the solution of the elliptic difference problem (EDP) in $L_{2 h}$.

Theorem 3.2. ([22]) For the solution of the EDP

$$
\left\{\begin{array}{l}
A_{h}^{x} u^{h}(x)=\omega^{h}(x), x \in \widetilde{\Omega}_{h}, \\
u^{h}(x)=0, x \in S_{h}
\end{array}\right.
$$

the coercivity estimate holds :

$$
\sum_{r=1}^{n}\left\|\left(u_{k}^{h}\right)_{\bar{x}_{r} \bar{x}_{r}, j_{r}}\right\|_{L_{2 h}} \leq M\left\|\omega^{h}\right\|_{L_{2 h}}
$$

where $M$ does not depend on $\omega^{h}$ and $h$.

Theorem 3.3. Solution of difference problem (3.3) satisfies the following coercivity SE:

$$
\left\|\left\{\tau^{-1}\left(u_{k}^{h}-u_{k-1}^{h}\right)\right\}_{1}^{N}\right\|_{\mathcal{C}_{1}^{\alpha}\left(L_{2 h}\right)} \leq M(\delta, \mu, \alpha)\left[\left\|\varphi^{h}\right\|_{W_{2 h}^{2}}+\frac{1}{\alpha(1-\alpha)}\left\|\left\{\psi_{k}^{h}\right\}_{1}^{N}\right\|_{\mathcal{C}_{1}^{\alpha}\left(W_{2 h}^{2}\right)}\right],
$$

where $M(\delta, \mu, \alpha)$ is independent of $\tau,\left\{\psi_{k}^{h}\right\}_{1}^{N}$, and $\varphi^{h}(x)$.

\section{Numerical examples}

4.1. 1D example. To illustrate test example, for one-dimensional RP equation and given functions 


$$
\begin{aligned}
& \varphi(x)=\left(e^{-1}+\frac{e^{-3}}{3}-\frac{1}{3}\right) \sin x, \mu(t)=e^{-2 t}, \\
& f(t, x)=-e^{-t}(4 \cos x+\cos 2 x), t \in[0,1], x \in[0, \pi],
\end{aligned}
$$

we consider the following BVP

$$
\left\{\begin{array}{l}
u_{t}(t, x)+(2+\cos x) u_{x x}(t, x)+\sin x u_{x}(t, x)-u(t, x)=f(t, x), \\
0 \leq t \leq 1, x \in(0, \pi), \\
u(1, x)=\int_{0}^{1} \mu(s) u(s, x) d s+\varphi(x), x \in[0, \pi], \\
u(t, 0)=0, u(t, \pi)=0, t \in[0,1]
\end{array}\right.
$$

with nonlocal integral condition.

Exact solution of problem(4.1) $u(t, x)=e^{-t} \sin x$.

Now, applying (3.3) to this problem, we get the next second order of ADS in $t$ and $x$ for approximation of BVP (4.1):

$$
\left\{\begin{array}{l}
\frac{u_{n}^{k}-u_{n}^{k-1}}{\tau \tau}+(1+\tau / 2) u_{n}^{k-1}+q_{1, n}\left(u_{n+1}^{k-1}-u_{n-1}^{k-1}\right)(2 h)^{-1} \\
+\frac{q_{2, n}}{h^{2}}\left(u_{n+1}^{k-1}-2 u_{n}^{k-1}+u_{n-1}^{k-1}\right)-\frac{q_{3, n}}{2 h^{3}}\left(u_{n+2}^{k-1}-2 u_{n+1}^{k-1}+2 u_{n-1}^{k-1}-u_{n-2}^{k-1}\right) \\
-\frac{q_{4, n}}{h^{4}}\left(u_{n+2}^{k-1}-4 u_{n+1}^{k-1}+6 u_{n}^{k-1}-4 u_{n-1}^{k-1}+u_{n-2}^{k-1}\right) \\
=-\left(1-\frac{\tau}{2}\right) e^{-t_{k-\frac{1}{2}}}\left(4 \cos x_{n}+\cos 2 x_{n}\right) \\
-\frac{\tau}{2}\left(\left(2+\cos \left(x_{n}\right)\right) e^{-t} k-\frac{1}{2}\left(-4 \cos x_{n}-4 \cos 2 x_{n}\right)\right. \\
\left.-\sin \left(x_{n}\right)\left(-4 \sin x_{n}-2 \sin 2 x_{n}\right)\right), t_{k-\frac{1}{2}}=t_{k}-\frac{\tau}{2}, n=\overline{2, M-2},, k=\overline{1, N} \\
u_{0}^{k}=0, u_{1}^{k}=\frac{4}{5} u_{2}^{k}-\frac{1}{5} u_{3}^{k}, u_{M}^{k}=0, u_{M-1}^{k}=\frac{4}{5} u_{M-2}^{k}-\frac{1}{5} u_{M-3}^{k}, k=\overline{0, N}, \\
u_{n}^{N}=\sum_{j=1}^{N} \frac{1}{2} \mu\left(t_{j}-\frac{\tau}{2}\right) \tau\left[u_{n}^{j}+u_{n}^{j-1}\right]+\varphi_{n}, n=\overline{0, M},
\end{array}\right.
$$

where

$$
\begin{aligned}
& q_{1, n}=\left(1+2 \tau+\tau \cos x_{n}\right) \sin x_{n}, \\
& q_{2, n}=\left(\left(2+\cos x_{n}\right)\left(1+\tau+\frac{3 \tau}{2} \cos x_{n}\right)-\tau \sin ^{2} x_{n}\right), \\
& q_{3, n}=\left(2+\cos x_{n}\right) \sin x_{n} \tau, q_{4, n}=\left(2+\cos \left(x_{n}\right)\right)^{2} .
\end{aligned}
$$

One can write (4.2) in the next matrix form

$$
\left\{\begin{array}{l}
A_{n} u_{n+2}+B_{n} u_{n+1}+C_{n} u_{n}+D_{n} u_{n-1}+E_{n} u_{n-2}=I_{N+1} \varphi_{n}, n=\overline{2, M-2} \\
u_{0}=\overrightarrow{0}, u_{1}=\frac{4}{5} u_{2}-\frac{1}{5} u_{3}, u_{M-1}=\frac{4}{5} u_{M-2}-\frac{1}{5} u_{M-3}, u_{M}=\overrightarrow{0}
\end{array}\right.
$$

Here, $I_{k}$ is $k \times k$ identity matrix, $\varphi_{n}$ is $(N+1) \times 1$ type matrix, and $A_{n}, B_{n}, C_{n}$, $D_{n}, E_{n}$ are $(N+1) \times(N+1)$ type matrices

$$
\varphi_{n}=\left[\begin{array}{lll}
\varphi_{n}^{0} & \cdots & \varphi_{n}^{N}
\end{array}\right]^{t},
$$


$O_{k \times m}$ is $k \times m$ type matrix with zero elements,

$$
\begin{aligned}
& A_{n}=\left[\begin{array}{c}
O_{1 \times(N+1)} \\
v_{n} I_{N}
\end{array}\right], O_{N \times 1}=\left[\begin{array}{c}
O_{1 \times(N+1)} \\
y_{n} I_{N}
\end{array}\right],
\end{aligned}
$$

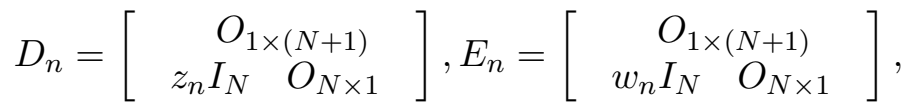

$$
\begin{aligned}
& C_{n}=\left[\begin{array}{ccccccc}
s_{0} & s_{1} & s_{2} & \cdots & s_{N-2} & s_{N-1} & s_{N} \\
r_{n} & d & 0 & \cdots & 0 & 0 & 0 \\
0 & r_{n} & d & \cdots & 0 & 0 & 0 \\
\vdots & \ddots & \ddots & \ddots & \vdots & \vdots & \vdots \\
0 & 0 & 0 & \ddots & \ddots & 0 & 0 \\
0 & 0 & 0 & \ddots & r_{n} & d & 0 \\
0 & 0 & 0 & \cdots & 0 & r_{n} & d
\end{array}\right]
\end{aligned}
$$

where

$$
\begin{aligned}
& v_{n}=\frac{\tau}{h^{3}} q_{3, n}-\frac{\tau}{2 h^{4}} q_{4, n}, d=\frac{1}{\tau}, y_{n}=\frac{1}{2 h} q_{1, n}+\frac{1}{h^{2}} q_{2, n}+\frac{1}{h^{3}} q_{3, n}, \\
& r_{n}=1+\frac{\tau}{2}-\frac{1}{\tau}-\frac{2}{h^{2}} q_{2, n}-\frac{3 \tau}{h^{4}} q_{4, n}, w_{n}=\frac{\tau}{2 h^{3}} q_{3, n}-\frac{\tau}{2 h^{4}} q_{4, n}, \\
& z_{n}=-\frac{1}{2 h} q_{1, n}+\frac{1}{h^{2}} q_{2, n}-\frac{\tau}{h^{3}} q_{3, n}+\frac{2 \tau}{h^{4}} q_{4, n}, s_{0}=-\frac{\tau}{2} \mu\left(\frac{\tau}{2}\right), \\
& s_{N}=1-\frac{\tau}{2} \mu\left(t_{N}-\frac{\tau}{2}\right), s_{j}=-\frac{\tau}{2}\left(\mu\left(t_{j-\frac{1}{2}}\right)+\mu\left(t_{j+\frac{1}{2}}\right)\right), j=1, \ldots, N-1 .
\end{aligned}
$$

A solution of (4.3) is defined by modified Gaus elimination method ([21]):

$$
\begin{aligned}
& u_{n}=\alpha_{n+1} u_{n+1}+\beta_{n+1} u_{n+2}+\gamma_{n+1}, \\
& \beta_{n+1}=-F_{n}^{-1} A_{n}, \alpha_{n+1}=-F_{n}^{-1}\left(B_{n}+D_{n} \beta_{n}+E_{n} \alpha_{n-1} \beta_{n}\right), \\
& \gamma_{n+1}=-F_{n}^{-1}\left(I_{N+1} \varphi_{n}-D_{n} \gamma_{n}-E_{n} \alpha_{n-1} \gamma_{n}-E_{n} \gamma_{n-1}\right), \\
& F_{n}=\left(C_{n}+D_{n} \alpha_{n}+E_{n} \beta_{n-1}+E_{n} \alpha_{n-1} \alpha_{n}\right)
\end{aligned}
$$

for $n=M-2, \cdots, 0$, where

$$
\begin{aligned}
& \gamma_{1}=\gamma_{2}=O_{(N+1) \times 1}, \alpha_{1}=\beta_{1}=O_{(N+1) \times(N+1)}, \\
& \alpha_{2}=\frac{4}{5} I_{N+1}, \beta_{2}=-\frac{1}{5} I_{N+1}, \\
& u_{M}=\overrightarrow{0}, D_{M}=\left(\beta_{M-2}+5 I_{N+1}\right)-\left(4 I_{N+1}-\alpha_{M-2}\right) \alpha_{M-1}, \\
& u_{M-1}=D_{M}^{-1}\left[\left(4 I_{N+1}-\alpha_{M-2}\right) \gamma_{M-1}-\gamma_{M-2}\right] .
\end{aligned}
$$

For different values of $(N, M)$ error computed by next formula

$$
E u_{M}^{N}=\max _{1 \leq k \leq N-1}\left(\sum_{n=1}^{M-1}\left(u\left(x_{n}, t_{k}\right)-u_{n}^{k}\right)^{2} h\right)^{\frac{1}{2}}
$$

and presented in Table 1. Numerical results which presented in Table 1 shows good agreement with theoretical stability results for solution of proposed DS to RP BVP with Dirichlet boundary and integral conditions. We observe that the scheme (4.2) has the second order convergence as it is expected to be.

Table 1. The errors between the exact solution of BVP (4.1) and the numerical solutions for different values of $\tau$ and $h$. 


\begin{tabular}{|l|l|}
\hline$(\mathbf{N}, \mathbf{M})$ & $E u_{M}^{N}$ \\
\hline$(10,10)$ & $5.63 \times 10^{-3}$ \\
\hline$(20,20)$ & $9.65 \times 10^{-4}$ \\
\hline$(40,40)$ & $2.27 \times 10^{-4}$ \\
\hline$(80,80)$ & $5.75 \times 10^{-5}$ \\
\hline$(160,160)$ & $1.46 \times 10^{-5}$ \\
\hline$(320,320)$ & $3.69 \times 10^{-6}$ \\
\hline
\end{tabular}

4.2. 2D example. Now, we consider BVP for two dimensional RP equation with integral condition

$$
\left\{\begin{array}{l}
u_{t}(t, x, y)+u_{x x}(t, x, y)+u_{y y}(t, x, y)-u(t, x, y)=f(t, x, y) \\
0<x, y<1,0 \leq t \leq 1 \\
u(1, x, y)=\int_{0}^{1} \mu(\gamma) u(\gamma, x, y) d \gamma+\varphi(x, y), 0 \leq x, y \leq 1 \\
u(t, 0, y)=0, u(t, 1, y)=0,0 \leq y \leq 1,0 \leq t \leq 1 \\
u(t, x, 0)=0, u(t, x, 1)=0,0 \leq x \leq 1,0 \leq t \leq 1
\end{array}\right.
$$

Here

$$
\begin{aligned}
& \mu(t)=\frac{1}{2 e^{2 t}}, \varphi(x)=\left(e^{-1}+\frac{e^{-3}}{6}-\frac{1}{6}\right) \xi(x, y), \\
& \xi(x, y)=\sin x \sin y, f(t, x, y)=-4 e^{-t} \xi(x, y)
\end{aligned}
$$

Exact solution of problem (4.4) is $u(t, x, y)=e^{-t} \xi(x, y)$.

Let us take

$$
\begin{aligned}
& x_{m}=(m-1) h, y_{n}=(n-1) h, t_{k}=(k-1) \tau, \\
& f_{m, n}^{k}=-4 e^{-\left(t_{k}-\frac{\tau}{2}\right)} \xi\left(x_{m}, y_{n}\right), k=\overline{0, N}, n, m=\overline{0, M} .
\end{aligned}
$$

By using (3.3) and Crank-Nicolson scheme, one can get the second order of ADS for approximately solution of BVP (4.4)

$$
\left\{\begin{array}{l}
\frac{u_{m, n}^{k}-u_{m, n}^{k-1}}{\tau}+\frac{u_{m+1, n}^{k}-2 u_{m, n}^{k}+u_{m-1, n}^{k}}{2 h^{2}}+\frac{u_{m, n+1}^{k}-2 u_{m, n}^{k}+u_{m, n-1}^{k}}{2 h^{2}}-\frac{1}{2} u_{m, n}^{k} \\
+\frac{u_{m+1, n}^{k-1}-2 u_{m, n}^{k-1}+u_{m-1, n}^{k-1}+\frac{u_{m, n+1}^{k-1}-2 u_{m, n}^{k-1}+u_{m, n-1}^{k-1}}{2 h^{2}}-\frac{1}{2} u_{m, n}^{k-1}=f_{m, n}^{k},}{2 h^{2}} \\
k=\overline{1, N-1}, n, m=\overline{1, M-1}, \overline{1, M-1}, k=\overline{1, N-1}, \\
u_{0, n}^{k}=0, u_{m, 0}^{k}=0, n=\overline{1, M-1}, m=\overline{1, M-1, M-1} . \\
u_{m, n}^{N}=\sum_{j=1}^{N} \frac{\tau}{2} \mu\left(t_{j}-\frac{\tau}{2}\right)\left[u_{m, n}^{j}+u_{m, n}^{j-1}\right]+\psi_{m, n}, n, m=\overline{1, M-1,}
\end{array}\right.
$$

One can write (4.5) in the matrix form

$$
\left\{\begin{array}{l}
A u_{n+1}+B u_{n}+C u_{n-1}=I_{K} f^{(n)}, n=\overline{1, M-1} \\
u_{0}=\overrightarrow{0}, u_{M}=\overrightarrow{0}
\end{array}\right.
$$


Here, $f^{(n)}, u_{n-1}, u_{n}, u_{n+1}$ are the $K \times 1$ column matrices such that $K=(N+1)(M+1)$,

$$
\begin{aligned}
& f^{(i)}=\left[\begin{array}{llllllllll}
f_{0, i}^{0} & \cdots & f_{0, i}^{N} & f_{1, i}^{0} & \cdots & f_{1, i}^{N} & \cdots & f_{M, i}^{0} & \cdots & f_{M, i}^{N}
\end{array}\right]^{t}, \\
& u_{i}=\left[\begin{array}{llllllllll}
u_{0, i}^{0} & \cdots & u_{0, i}^{N} & u_{1, i}^{0} & \cdots & u_{1, i}^{N} & \cdots & u_{M, i}^{0} & \cdots & u_{M, i}^{N}
\end{array}\right]^{t}, \\
& i=n-1, n, n+1, a=\frac{1}{2 h^{2}}, b=-\frac{1}{\tau}-\frac{1}{2 h^{2}}-\frac{1}{2}, c=\frac{1}{\tau}-\frac{1}{2 h^{2}}-\frac{1}{2}, \\
& D=\left[\begin{array}{ccccc}
b & c & \cdots & 0 & 0 \\
0 & b & \ddots & 0 & 0 \\
\vdots & \ddots & \ddots & \ddots & \vdots \\
0 & 0 & \cdots & b & c \\
s_{0} & s_{1} & \cdots & s_{N-1} & s_{N}
\end{array}\right], E=\left[\begin{array}{ccccc}
a & a & \cdots & 0 & 0 \\
0 & a & \ddots & 0 & 0 \\
\vdots & \ddots & \ddots & \ddots & \vdots \\
0 & 0 & \cdots & a & a \\
0 & 0 & \cdots & 0 & 0
\end{array}\right], \\
& B=\left[\begin{array}{ccccccc}
I_{K} & O & O & \cdots & O & O & O \\
E & D & E & \cdots & O & O & O \\
O & E & D & \ddots & O & O & O \\
\vdots & \vdots & \ddots & \ddots & \ddots & \vdots & \vdots \\
\vdots & \vdots & \ddots & \ddots & \ddots & \ddots & \vdots \\
O & O & O & \cdots & E & D & E \\
O & O & O & \cdots & O & O & I_{K}
\end{array}\right], A=C=\operatorname{diag}\{O, E, \ldots E, O\} .
\end{aligned}
$$

A solution of (4.6) is defined by modified Gaus elimination method ([21]):

$$
\begin{aligned}
& u_{M}=\overrightarrow{0}, u_{n}=\alpha_{n} u_{n+1}+\beta_{n}, n=M-1, \cdots, 1, \\
& \alpha_{n}=-\left(B+C \alpha_{n-1}\right)^{-1} A, \beta_{n}=\left(B+C \alpha_{n-1}\right)^{-1}\left(I_{n} f^{(n)}-C \beta_{n-1}\right), \\
& \beta_{1}=O_{K \times 1}, \alpha_{1}=O_{K \times K} .
\end{aligned}
$$

The errors between the exact solution of (4.4) and the numerical solutions are calculated by

$$
E u_{M, M}^{N}=\max _{1 \leq k \leq N-1}\left(\sum_{m=1}^{M-1} \sum_{n=1}^{M-1}\left(u\left(x_{m}, y_{n}, t_{k}\right)-u_{m, n}^{k}\right)^{2} h^{2}\right)^{\frac{1}{2}} .
$$

Table 2. The errors between the exact solution of (4.4) and the numerical solutions for different values of $\tau$ and $h$.

\begin{tabular}{|l|l|}
\hline$(\mathbf{N}, \mathbf{M})$ & $E u_{M, M}^{N}$ \\
\hline$(5,5)$ & $1.90 \times 10^{-2}$ \\
\hline$(10,10)$ & $4.74 \times 10^{-3}$ \\
\hline$(20,20)$ & $1.18 \times 10^{-3}$ \\
\hline$(40,40)$ & $2.96 \times 10^{-4}$ \\
\hline
\end{tabular}

We observe that the scheme (4.5) has the second order convergence as it is expected to be. 


\section{Conclusion}

In this paper, we use finite difference method to find approximate solution of the RP problem with nonlocal integral condition. We give the second order of ADS for the approximation of RP problem. Proposed DS uses $A$ and $A^{2}$ and it is good for smooth input data for BVPs. We prove stability and coercive stability inequalities for solution of this DS. Later, we describe the second order of ADS for RP multidimensional problem with Dirichlet boundary condition and establish stability inequalities for solution. Lastly, by using MATLAB program, we give numerical illustration for simple $1 \mathrm{D}$ and $2 \mathrm{D}$ test problems. Notice that $1 \mathrm{D}$ test example is used second order of accuracy DS with $A^{2}$ term but $2 \mathrm{D}$ test example is carried out by using Crank-Nicolson DS which has the same order accuracy. It is well-known that Crank-Nicolson DS is good for realization but does not work with unsmooth data. In future work it could be established well-posedness such type DSs.

\section{References}

[1] R. K. M. Aldulaimi, An innovative receiver design for a parabolic trough solar collector using overlapped and reverse flow: An Experimental Study, Arabian Journal for Science and Engineering 44(9) (2019), 7529-7539.

[2] A. Ashyralyev, A. Dural and Y. Sozen, Multipoint nonlocal boundary value problems for reverse parabolic equations: well-posedness, Vestnik of Odessa National University: Mathematics and Mechanics 13 (2008), 1-12.

[3] A. Ashyralyev, A. Dural and Y. Sozen, Well-posedness of the Rothe difference scheme for reverse parabolic equations, Iran J. Optim. 1 (2009), 1-25.

[4] A. Ashyralyev, A. Dural and Y. Sozen, On well-posedness of the second order accuracy difference scheme for reverse parabolic equation, Malaysian Journal of Mathematical Sciences 6(S) (2012), 91-109

[5] A. Ashyralyev and P. E. Sobolevskii, Well-Posedness of Parabolic Difference Equations, Birkhäuser, Basel, Switzerland, 1994.

[6] C. Ashyralyyev, Well-posedness of boundary value problems for reverse parabolic equation with integral condition, e-Journal of Analysis and Applied Mathematics 2018(1) (2018), 11-20.

[7] C. Ashyralyyev, Stability of Rothe difference scheme for the reverse parabolic problem with integral boundary condition, Math.Meth.Appl. Sci.43 (2020), 5369-5379.

[8] C. Ashyralyyev, P.Akkan, Source identification problem for a parabolic equation with multipoint nonlocal boundary condition, Numerical Functional Analysis and Optimization, (2020), doi: 10.1080/01630563.2020.1802748, 1-23.

[9] C. Ashyralyyev, A. Dural and Y. Sozen, Finite difference method for the reverse parabolic problem, Abstr. Appl. Anal. 2012 (2012), 1-17.

[10] C. Ashyralyyev, A. Dural and Y. Sozen, Finite difference method for the reverse parabolic problem with Neumann condition, AIP Conference Proceedings 1470 (2012), $102-105$.

[11] D.N. Hao, N.V. Duc and N.V. Thang, Backward semi-linear parabolic equations with time-dependent coefficients and local Lipschitz source, Inverse Problems 34(5) (2018), Article Number: 055010.

[12] D. Ijacu and M. Marinescu, Filtering for non-markovian SDEs involving nonlinear SPDEs and backward parabolic equations, Applied Mathematics and Optimization, 70(3) 2014, 395-409. 
[13] T. Klimsiak, Strong solutions of semilinear parabolic equations with measure data and generalized backward stochastic differential equation, Potential Analysis, 36(2) (2012) 373-404.

[14] S. G. Krein, Linear Differential Equations in Banach Space, Nauka, Moscow, Russia, 1966.

[15] A. I. Kozhanov, Parabolic equations with unknown time-dependent coefficients, Comput. Math. Math. Phys. 57(6) (2017), 961-972.

[16] A. Lachapelle, J. Salomon and G. Turinici, Computation of mean field equilibria in economics, Mathematical Models 83 Methods in Applied Sciences, 20(4) (2010), $567-588$.

[17] T. LaRosa, The propagation of an electron beam through the solar corona, Ph.D. thesis, Department of Physics and Astronomy, University of Maryland, 1986.

[18] J. Martin-Vaquero, Two-level fourth-order explicit schemes for diffusion equations subject to boundary integral specifications, Chaos Solitons Fractals 42 (2009), 23642372 .

[19] J. Martin-Vaquero and J. Vigo-Aguiar, On the numerical solution of the heat conduction equations subject to nonlocal conditions, Appl. Numer.Math. 59 (2009), 2507-2514.

[20] S. G. Pyatkov and M. L. Samkov, Solvability of some inverse problems for the nonstationary heat-and-mass-transfer system, J. Math.Anal.Appl. 446(2) (2017), $1449-1465$.

[21] A. A. Samarskii, The Theory of Difference Schemes, New York, Dekker, 2001.

[22] P. E. Sobolevskii, Difference methods for the approximate solution of differential equations, Voronezh State University Press,Voronezh, Russia, 1975.

[23] K. Stewartson, Multistructural boundary layers on flat plates and related bodies, Advances in Applied Mechanics 14 (1974), 145-239.

[24] K. Stewartson, D'Alembert's paradox, SIAM Review, 23(3) (1981), 308-343.

[25] B.L.T. Thanh, F. Smarrazzo and A. Tesei, Sobolev regularization of a class of forward backward-parabolic equations, Journal of Differential Equations, 257(5) (2014), 1403-1456

[26] A.G.Yagola and M.V. Klibanov, Convergent numerical methods for parabolic equations with reversed time via a new Carleman estimate, Inverse Problems, 35(11) (2019), Article Number 115012.

Charyyar Ashyralyyev

Gumushane University, Faculty of Engineering and Natural Sciences,Department of Mathematical Engineering, Gumushane, 29100, Turkey, TAU, Department of Computer Technology, Ashgabat, 744000, Turkmenistan.

E-mail address: charyar@gmail.com

Received: April 3, 2020; Revised: September 13, 2020; Accepted: October 30, 2020 